\title{
THE RIESZ POTENTIAL IN GENERALIZED ORLICZ SPACES
}

\author{
PETTERI HARJULEHTO AND PETER HÄSTÖ
}

\begin{abstract}
In this article we prove a Riesz potential estimate and a Sobolev inequality for general generalized Orlicz spaces. Our assumptions are natural generalizations of the log-Hölder continuity that is commonly used in the variable exponent case. We also provide a number of useful auxiliary results including a normalization of the $\Phi$-function and behavior under duality.
\end{abstract}

\section{INTRODUCTION}

Generalized Orlicz spaces $L^{\varphi(\cdot)}$ have been studied since the 1940's. A major synthesis of functional analysis in these spaces is given in the monograph of Musielak [26] from 1983 and so the spaces have also been called Musielak-Orlicz spaces. These spaces are similar to Orlicz spaces, but defined by a more general function $\varphi(x, t)$ which may vary with the location in space: the norm is defined by means of the integral

$$
\int_{\mathbb{R}^{n}} \varphi(x,|f(x)|) d x
$$

whereas in an Orlicz spaces $\varphi$ would be independent of $x, \varphi(|f(x)|)$. In the special case $\varphi(t)=t^{p}$ we obtain the Lebesgue space $L^{p}$.

Minimization problems in the calculus of variations have had a similar course of generalization (e.g. [15, 24]): from

$$
\min _{u} \int|\nabla u|^{2} d x \text { to } \min _{u} \int|\nabla u|^{p} d x \quad \text { to } \min _{u} \int \varphi(x,|\nabla u|) d x .
$$

Usually, the function $\varphi$ is assumed to have $p$-growth conditions, i.e. $\varphi(x, t) \approx t^{p}$ uniformly. This restriction means that the full complexity of the minimization problem is avoided.

The special case $\varphi(x, t):=t^{p(x)}$, so-called variable exponent spaces $L^{p(\cdot)}$, and corresponding differential equations with non-standard growth have been vigorously studied in recent years [9, 12, 18]. The spaces were introduced by Orlicz already in 1931 [29], but the field lay dormant for a long time. Some 70 years later, key results in harmonic analysis (e.g., [10, 11, 27]) and regularity theory (e.g., [1, 8]) were established.

The reason that variable exponent spaces thrived while little was done in generalized Orlicz spaces was the belief that many results from Lebesgue and Sobolev spaces can be obtained in the former setting but not the latter. However, this belief has been challenged recently, based on new techniques that were developed and perfected in the context of variable exponent spaces.

Date: November 7, 2015.

2010 Mathematics Subject Classification. 46E30; 42B20.

Key words and phrases. Generalizer Orlicz space, Musielak-Orlicz spaces, Riesz potential, Sobolev embedding, variable exponent, non-standard growth. 
In addition to being a natural generalization which covers results from both variable exponent and Orlicz spaces, the study of generalized Orlicz spaces can be motivated by applications to differential equations, image processing and fluid dynamics.

Chen, Levine and Rao [6] introduced a model for image restoration based on a particular type of generalized $\Phi$-function:

$$
\varphi(x, t)= \begin{cases}\frac{1}{q(x)} t^{q(x)}, & \text { when } t \leqslant \beta, \\ t-\beta+\frac{1}{q(x)} \beta^{q(x)}, & \text { when } t \geqslant \beta .\end{cases}
$$

Since they only consider a bounded domain, the space needed actually turns out to be $L^{1}$ (or, more precisely, $B V$ ), see Proposition 4.2. In [17] we analyzed the $L^{p(\cdot)}$-variant of this model. More recently, Alaouia, Nabilab and Altanjia [2] have considered a general structure PDE in the image processing context, but again work in $B V$.

Wróblewska-Kamińska [31] has studied fluid dynamics models with generalized Orlicz-type structure conditions, and Świerczewska-Gwiazda [30] studied existence of solutions to parabolic equations with generalized Orlicz growth. Giannetti and Passarelli di Napoli [14] and Baroni, Colombo and Mingione [3, 4, 7] studied the regularity of solutions to the minimization problems

$$
\min _{u} \int|\nabla u|^{p(x)} \log (e+|\nabla u|) d x \quad \text { and } \min _{u} \int|\nabla u|^{p}+a(x)|\nabla u|^{q} d x,
$$

respectively. The regularity of minimizers depends on the regularity of the exponents $p$ and $q$, and the weight $a$.

Giannetti and Passarelli di Napoli studied a very special form of functional. Also in the function space setting the first steps from $L^{p(\cdot)}$ were $\Phi$-functions of type $t^{p(\cdot)} \log (e+$ $t)^{q(\cdot)}$ which were studied in several papers, e.g., [21, 25]. Hopefully, the tools presented in this paper will allow the research community to bypass the stage of special log-type variants in the study of PDE and move directly to the general form, including, among others, those studied by Colombo and Mingione.

A key tool for harmonic analysis is the (Hardy-Littlewood) maximal operator $M$. Maeda, Mizuta, Ohno and Shimomura [22, 23, 28] were first to study it in $L^{\varphi(\cdot)}$, with somewhat heavy machinery. Their result on the boundedness of $M$ was generalized by Hästö $[19,20]$ by removing unnecessary assumptions and simplifying the proof.

The Sobolev embedding has been studied in generalized Orlicz spaces by Fan [13]. He uses a reduction to the $W^{1,1}$-case based on direct differentiation of the $\Phi$-function. This leads to extraneous assumptions concerning the derivative $\varphi^{\prime}$. In this paper we prove the Sobolev embedding by Hedberg's method, establishing the boundedness of the Riesz potential. A similar approach was used in [22], however, that paper contains several complicated assumptions and a target spaces which is not explicitly defined (cf. page 91 of the reference). Our proof is more versatile and requires fewer assumptions than the previously known ones, and provide a new perspective even in Orlicz spaces. We hope that our simple and clear results and techniques will allow most of the results that have been derived in $L^{p(\cdot)}$ over the past 15 years to be established in $L^{\varphi(\cdot)}$ as well.

\section{BACKGROUND}

The notation $f \lesssim g$ means that there exists a constant $C>0$ such that $f \leqslant C g$. The notation $f \approx g$ means that $f \lesssim g \lesssim f$. The space $A \cap B$ is endowed with the norm $\|f\|_{A \cap B}=\max \left\{\|f\|_{A},\|f\|_{B}\right\}$. For a real function $f$ we denote

$$
f\left(x^{-}\right):=\lim _{\varepsilon \rightarrow 0^{+}} f(x-\varepsilon) \text { and } f\left(x^{+}\right):=\lim _{\varepsilon \rightarrow 0^{+}} f(x+\varepsilon) .
$$


By $L^{0}\left(\mathbb{R}^{n}\right)$ we denote the set of (Lebesgue) measurable functions on $\mathbb{R}^{n}$. The (Hardy-Littlewood) maximal operator is defined for $f \in L^{0}\left(\mathbb{R}^{n}\right)$ by

$$
M f(x):=\sup _{r>0} f_{B(x, r)}|f(y)| d y,
$$

where $B(x, r)$ is the open ball with center $x$ and radius $r$, and $f$ denotes the average integral.

We recall some definitions pertaining to generalized Orlicz spaces. For proofs and further properties see [12, Chapter 2] and [26].

Definition 2.1. A convex function $\varphi \in C([0, \infty) ;[0, \infty])$ with $\varphi(0)=\varphi\left(0^{+}\right)=0$, and $\lim _{t \rightarrow \infty} \varphi(t)=\infty$ is called a $\Phi$-function. The set of $\Phi$-functions is denoted by $\Phi$.

Instead of the usual left-continuity, we have assumed that every $\Phi$-function is continuous in the compactification $[0, \infty]$. This is not a restriction as every function satisfying the former condition is equivalent to one satisfying the latter, see [16]. Recall that two functions $\varphi$ and $\psi$ are equivalent, $\varphi \simeq \psi$, if there exists $L \geqslant 1$ such that $\psi\left(\frac{t}{L}\right) \leqslant \varphi(t) \leqslant \psi(L t)$ for relevant all $t$. Equivalent $\Phi$-functions give rise to the same space with comparable norms.

Note that every $\Phi$-function is increasing on $[0, \infty)$ and strictly increasing on $\{x$ : $\varphi(x) \in(0, \infty)\}$. By $\varphi^{-1}$ we denote the left-continuous inverse of $\varphi \in \Phi$,

$$
\varphi^{-1}(\tau):=\inf \{t \geqslant 0: \varphi(t) \geqslant \tau\} .
$$

It follows directly from this definition that $\varphi^{-1}(\varphi(t)) \leqslant t$ and equality holds if $\varphi$ is strictly increasing. To be more precise, if $t_{0}:=\max \{t \mid \varphi(t)=0\}$ and $t_{\infty}:=$ $\max \{t \mid \varphi(t)<\infty\}$, then

$$
\varphi^{-1}(\varphi(t))= \begin{cases}0, & t \leqslant t_{0} \\ t, & t_{0}<t \leqslant t_{\infty} \\ t_{\infty} & t \geqslant t_{\infty} .\end{cases}
$$

Note that $\varphi^{-1}(\varphi(t))=t$ if $\varphi(t) \in(0, \infty)$. In the opposite order thing work better, since the continuity of $\varphi$ implies that

$$
\varphi\left(\varphi^{-1}(s)\right)=s .
$$

Note that $\varphi \simeq \psi$ if and only if $\varphi^{-1} \approx \psi^{-1}$.

If $\varphi \approx \psi$, then by convexity $\varphi \simeq \psi$ We say that $\varphi$ is doubling if $\varphi(2 t) \leqslant A \varphi(t)$ for every $t>0$. For a doubling $\Phi$-function $\simeq$ and $\approx$ are equivalent. A $\Phi$-function can be represented as

$$
\varphi(t)=\int_{0}^{t} \varphi^{\prime}(s) d s
$$

in the set $\{\varphi(t)<\infty\}$, where $\varphi^{\prime}$ is the right-continuous right-derivative of the convex function $\varphi$.

Definition 2.4. The set $\Phi\left(\mathbb{R}^{n}\right)$ consists of those $\varphi: \mathbb{R}^{n} \times[0, \infty) \rightarrow[0, \infty]$ with

(1) $\varphi(y, \cdot) \in \Phi$ for every $y \in \mathbb{R}^{n}$; and

(2) $\varphi(\cdot, t) \in L^{0}\left(\mathbb{R}^{n}\right)$ for every $t \geqslant 0$.

Also the functions in $\Phi\left(\mathbb{R}^{n}\right)$ will be called $\Phi$-functions. In sub- and superscripts the dependence on $x$ will be emphasized by $\varphi(\cdot): L^{\varphi}$ (Orlicz) vs $L^{\varphi(\cdot)}$ (generalized Orlicz). 
Properties and definitions of $\Phi$-functions carry over to generalized $\Phi$-functions pointwise. In particular,

$$
\varphi^{-1}(x, \tau):=\inf \{t \geqslant 0: \varphi(x, t) \geqslant \tau\}
$$

is the left-continuous inverse with respect to the second parameter.

Definition 2.5. Let $\varphi \in \Phi\left(\mathbb{R}^{n}\right)$ and define the modular $\varrho_{\varphi(\cdot)}$ for $f \in L^{0}\left(\mathbb{R}^{n}\right)$ by

$$
\varrho_{\varphi(\cdot)}(f):=\int_{\mathbb{R}^{n}} \varphi(x,|f(x)|) d x .
$$

The generalized Orlicz space, also called Musielak-Orlicz space, is defined as the set

$$
L^{\varphi(\cdot)}\left(\mathbb{R}^{n}\right)=\left\{f \in L^{0}\left(\mathbb{R}^{n}\right): \lim _{\lambda \rightarrow 0} \varrho_{\varphi(\cdot)}(\lambda f)=0\right\}
$$

equipped with the (Luxemburg) norm

$$
\|f\|_{\varphi(\cdot)}:=\inf \left\{\lambda>0: \varrho_{\varphi(\cdot)}\left(\frac{f}{\lambda}\right) \leqslant 1\right\} .
$$

Auxiliary results in generalized Orlicz spaces. A problem when modifying $\Phi$-functions is that we easily move out of the domain of convex functions. The next lemma often allows us to rectify this.

Lemma 2.6 (Lemma 3.1, [19]). Let $\varphi:[0, \infty) \rightarrow[0, \infty]$ be a left-continuous function with $\varphi(0)=\varphi\left(0^{+}\right)=0$, and $\lim _{t \rightarrow \infty} \varphi(t)=\infty$. If $s \mapsto \frac{\varphi(s)}{s}$ is increasing, then $\varphi$ is equivalent to a convex function $\psi \in \Phi$.

Define $\varphi_{B}^{-}(t):=\inf _{x \in B} \varphi(x, t)$ and $\varphi_{B}^{+}(t):=\sup _{x \in B} \varphi(x, t)$. We state three assumptions which together imply the boundedness of the maximal operator [19, 20].

(A0M) There exists $\beta>0$ such that $\varphi(x, \beta) \leqslant 1$ and $\varphi(x, 1) \geqslant 1$ for all $x \in \mathbb{R}^{n}$.

(A1M) There exists $\beta \in(0,1)$ such that

$$
\varphi_{B}^{+}(\beta t) \leqslant \varphi_{B}^{-}(t)
$$

for every $t \in\left[1,\left(\varphi_{B}^{-}\right)^{-1}\left(\frac{1}{|B|}\right)\right]$ and every ball $B$ with $1 /|B| \geqslant \varphi_{B}^{-}(1)$.

(A2M) There exists $\beta>0$ and $h \in L^{1}\left(\mathbb{R}^{n}\right) \cap L^{\infty}\left(\mathbb{R}^{n}\right)$ such that, for every $t \in[0,1]$,

$$
\varphi(x, \beta t) \lesssim \varphi(y, t)+h(x)+h(y) .
$$

Theorem 2.7 (Theorem 4.7, [19, 20]). Let $\varphi \in \Phi\left(\mathbb{R}^{n}\right)$ satisfy assumptions (AOM)$(A 2 M)$, and assume that there exists $\gamma>1$ such that $s \mapsto s^{-\gamma} \varphi(x, s)$ is increasing for every $x \in \mathbb{R}^{n}$. Then

$$
M: L^{\varphi(\cdot)}\left(\mathbb{R}^{n}\right) \rightarrow L^{\varphi(\cdot)}\left(\mathbb{R}^{n}\right)
$$

is bounded.

Note that the assumption that $s \mapsto s^{-\gamma} \varphi(x, s)$ is increasing, is a natural generalization of the Lebesgue space condition $p>1$.

Remark 2.8. Some examples of generalized $\Phi$-functions:

$$
\varphi_{1}(x, t)=t^{p(x)} \log (1+t), \quad \varphi_{2}(x, t)=t^{p}+a(x) t^{q}, \quad \varphi_{3}(x, t) \quad=e^{p(x) t}-1 .
$$

The first and second $\Phi$-functions have been recently studied in [7, 14], while $\varphi_{3}$ is an example of a non-doubling $\Phi$-function.

The boundedness of the maximal operator in $[19,20]$ covers all of them, as do the auxiliary results in this paper, including normalization and duality. For the Riesz potential we need to assume that $t^{\frac{\varepsilon-n}{\alpha}} \varphi(t)$ is decreasing. This is a natural generalization 
of the Lebesgue space condition $p<n$, and it implies that $\varphi$ is doubling (with constant $\left.2^{\frac{n-\varepsilon}{\alpha}}\right)$.

\section{THE ASSUMPTIONS}

For our study of generalized Orlicz spaces, we need three main assumptions, which are variants of $(\mathrm{AxM})$ from Section 2.

(A0) $\varphi^{-1}(x, 1) \approx 1$.

(A1) There exists $\beta \in(0,1)$ such that $\beta \varphi^{-1}(x, t) \leqslant \varphi^{-1}(y, t)$ for every $t \in\left[1, \frac{1}{|B|}\right]$, every $x, y \in B$ and every ball $B$ with $|B| \leqslant 1$.

(A2) $L^{\varphi(\cdot)}\left(\mathbb{R}^{n}\right) \cap L^{\infty}\left(\mathbb{R}^{n}\right)=L^{\varphi_{\infty}}\left(\mathbb{R}^{n}\right) \cap L^{\infty}\left(\mathbb{R}^{n}\right)$, with $\varphi_{\infty}(t):=\limsup _{|x| \rightarrow \infty} \varphi(x, t)$.

In this section we elaborate on these and add some technical details. Recalling that $\varphi \simeq \psi$, if and only if $\varphi^{-1} \approx \psi^{-1}$, we establish the following invariance.

Lemma 3.1. These assumptions are invariant under equivalence of $\Phi$-functions, i.e. if $\varphi \simeq \psi$, then $\varphi$ satisfies $(A x)$ if and only if $\psi$ does.

We convert in three steps the original $\varphi$ function to an equivalent $\Phi$-function $\bar{\varphi}$ which is more regular. Let us next investigate each assumption in turn.

Assumption (A0). First we study relations between (A0M) and (A0).

Lemma 3.2. Assumption (AOM) implies (AO).

Proof. By the definition of $\varphi^{-1}$, the inequality $\varphi(x, 1) \geqslant 1$ yields $\varphi^{-1}(x, 1) \leqslant 1$. If $\varphi(x, \beta)<1$, then $\varphi^{-1}(x, 1) \geqslant \beta$. If $\varphi(x, \beta)=1$, then by convexity $\varphi(x, \beta / 2)<1$ and thus $\varphi^{-1}(x, 1) \geqslant \beta / 2$.

The converse is not true. If (A0) holds, so that $c_{1} \leqslant \varphi^{-1}(x, 1) \leqslant c_{2}$, then $\varphi\left(x, c_{1}\right) \leqslant$ 1 and $\varphi\left(x, c_{2}\right) \geqslant 1$. But it is not necessary that $\varphi(x, 1) \geqslant 1$ as the following example shows: if $\varphi(t):=t^{2} / 2$, then $\varphi(x, 1)=\frac{1}{2}<1$ but $\varphi^{-1}(x, 1)=2$.

We use the assumption (A0) to find an equivalent $\Phi$-function that behaves better than the original one. We set

$$
\varphi_{1}(x, t):=\varphi\left(x, \varphi^{-1}(x, 1) t\right) .
$$

Then $\varphi_{1}$ is equivalent to $\varphi$ and $\varphi_{1}^{-1}(x, 1) \equiv \varphi_{1}(x, 1) \equiv 1$ (by (2.3)). The set of $\Phi$-functions with $\varphi^{-1}(x, 1) \equiv 1$ will be denoted $\Phi_{1}\left(\mathbb{R}^{n}\right)$. Note that every $\Phi_{1}\left(\mathbb{R}^{n}\right)$ function satisfies assumptions (A1) and (A0M).

Assumption (A1). Let us start by reformulating (A1) when $\varphi \in \Phi_{1}\left(\mathbb{R}^{n}\right)$.

Lemma 3.3. Let $\varphi \in \Phi_{1}$. Condition (A1) holds if and only if there exists $\beta>0$ such that

$$
\varphi(x, \beta t) \leqslant \varphi(y, t)
$$

for every $t \in\left[1, \varphi^{-1}\left(y, \frac{1}{|B|}\right)\right]$, every $x, y \in B$ and every ball $B$ with $|B| \leqslant 1$.

Proof. Let the condition of the lemma hold and assume $t \in\left[1, \frac{1}{|B|}\right]$. Then $\varphi^{-1}(y, t) \in$ $\left[1, \varphi^{-1}\left(y, \frac{1}{|B|}\right)\right]$ and so

$$
\varphi\left(x, \beta \varphi^{-1}(y, t)\right) \leqslant \varphi\left(y, \varphi^{-1}(y, t)\right)=t .
$$

Let $t_{0}$ and $t_{\infty}$ be as in (2.2) and abbreviate $s:=\beta \varphi^{-1}(y, t)$. If $s \in\left(t_{0}, t_{\infty}\right]$, then (A1) follows from the previous inequality, since $\varphi^{-1}(x, \varphi(x, s))=s$. And if $s>t_{\infty}$, then 
$\varphi(x, s)=\infty \leqslant t$, a contradiction, so this is not possible. If $s \leqslant t_{0}$, then $s \leqslant \varphi^{-1}(x, t)$ since $\varphi^{-1}(x, t)>t_{0}(t>0)$. Thus in each case (A1) holds.

Assume then that (A1) holds and let $t \in\left[1, \frac{1}{|B|}\right]$. By (A1) and (2.3),

$$
\varphi\left(x, \beta \varphi^{-1}(y, t)\right) \leqslant \varphi\left(x, \varphi^{-1}(x, t)\right)=t=\varphi\left(y, \varphi^{-1}(y, t)\right) .
$$

Let $s:=\varphi^{-1}(y, t)$. Thus $\varphi(x, \beta s) \leqslant \varphi(y, s)$ in the range of $\varphi^{-1}(y, \cdot)$, including $\left(t_{0}, t_{\infty}\right)$. When $s \rightarrow t_{0}^{+}$, this gives that $\varphi\left(x, \beta t_{0}\right) \leqslant \varphi\left(y, t_{0}\right)=0$, so the inequality holds for $s \leqslant t_{0}$, as well. Finally, if $s \geqslant t_{\infty}$, then $\varphi(y, s)=\infty$, so the inequality certainly holds.

Corollary 3.4. If $\varphi \in \Phi_{1}\left(\mathbb{R}^{n}\right)$ satisfies (A1), then it satisfies assumption (A1M).

Proof. Let $B$ be a ball with $|B| \leqslant 1$. We must show that $\varphi_{B}^{+}(\beta t) \leqslant \varphi_{B}^{-}(t)$ when $t \in\left[1,\left(\varphi_{B}^{-}\right)^{-1}\left(\frac{1}{|B|}\right)\right]$.

Suppose first that $t$ is not the upper end-point of the interval. For such $t$, there exist $y_{i} \in B$ such that $t \in\left[1, \varphi^{-1}\left(y_{i}, \frac{1}{|B|}\right)\right]$ and $\varphi_{B}^{-}(t)=\lim _{j} \varphi\left(y_{j}, t\right)$. Then by Lemma 3.3

$$
\varphi(x, \beta t) \leqslant \varphi\left(y_{j}, t\right) .
$$

We let $j \rightarrow \infty$ and take the supremum over $x \in B$ to arrive at (A1M).

It remains to consider $t=\left(\varphi_{B}^{-}\right)^{-1}\left(\frac{1}{|B|}\right)$. Suppose first that $\varphi_{B}^{+}(\beta t)<\infty$. Let $\varepsilon>0$ and choose $x \in B$ such that $\varphi_{B}^{+}(\beta t) \leqslant(1+\varepsilon) \varphi(x, \beta t)$. Since $\varphi(x, \cdot)$ is left-continuous, we can choose $t^{\prime}<t$ such that $\varphi(x, \beta t) \leqslant(1+\varepsilon) \varphi\left(x, \beta t^{\prime}\right)$. Combining this with the previous case, we obtain that

$$
\varphi_{B}^{+}(\beta t) \leqslant(1+\varepsilon)^{2} \varphi\left(x, \beta t^{\prime}\right) \leqslant(1+\varepsilon)^{2} \varphi\left(y, t^{\prime}\right) \leqslant(1+\varepsilon)^{2} \varphi(y, t) .
$$

Taking infimum over $y$ and letting $\varepsilon \rightarrow 0$, we obtain the desired inequality. The case $\varphi_{B}^{+}(\beta t)=\infty$ is handled analogously.

Remark 3.5. When $\varphi(x, t)=t^{p(x)}$, (A1) corresponds to the local log-Hölder continuity condition of $\frac{1}{p}$. Namely let $x, y \in \mathbb{R}^{n}$ with $|x-y| \leqslant \frac{1}{2}$. Let $B$ be such a ball that $x, y \in$ $B$ and $\operatorname{diam}(B)=2|x-y|$. By symmetry, we may assume that $p(x)<p(y)$. Since $\varphi^{-1}(x, t)=t^{1 / p(x)}$, assumption (A1) reads $\beta\left(\omega_{n}|x-y|^{n}\right)^{-1 / p(x)} \leqslant\left(\omega_{n}|x-y|^{n}\right)^{-1 / p(y)}$, where $\omega_{n}$ is the measure of the unit ball. In other words,

$$
\left(\omega_{n}^{-1}|x-y|^{-n}\right)^{\frac{1}{p(x)}-\frac{1}{p(y)}} \leqslant \frac{1}{\beta} .
$$

Taking the logarithm, we find that

$$
\frac{1}{p(x)}-\frac{1}{p(y)} \leqslant \frac{\log \frac{1}{\beta}}{n \log \left(|x-y|^{-1}\right)-\log \omega_{n}} \lesssim \frac{1}{\log \left(e+|x-y|^{-1}\right)} .
$$

Assumption (A2). Again, the assumption $\varphi \in \Phi_{1}\left(\mathbb{R}^{n}\right)$ allows us to reformulate (A2).

Lemma 3.6. Let $\varphi \in \Phi_{1}$. If $\varphi$ satisfies (A2), then it satisfies (A2M).

Proof. By Theorem 2.8.1 of [12], $L^{\psi(\cdot)}\left(\mathbb{R}^{n}\right) \subset L^{\varphi(\cdot)}\left(\mathbb{R}^{n}\right)$ if and only if there exist $\beta>0$ and $h \in L^{1}\left(\mathbb{R}^{n}\right)$ such that $\varphi(x, \beta t) \leqslant \psi(x, t)+h(x)$. Hence (A2) implies that there exists $\beta$ and $h \in L^{1}\left(\mathbb{R}^{n}\right)$ such that

$$
\varphi(x, \beta t) \leqslant \varphi_{\infty}(t)+h(x) \text { and } \varphi_{\infty}(\beta t) \leqslant \varphi(x, t)+h(x)
$$

for all $t \in[0,1]$ (the restricted range of $t$ is due to the intersection with $L^{\infty}\left(\mathbb{R}^{n}\right)$ in the assumption). From these we obtain that

$$
\varphi\left(x, \beta^{2} t\right) \leqslant \varphi_{\infty}(\beta t)+h(x) \leqslant \varphi(y, t)+h(y)+h(x)
$$


for $t \in[0,1]$. Since $\varphi(x, 1) \equiv 1, \varphi\left(x, \beta^{2} t\right) \leqslant 1$. So the inequality also holds when we replace $h$ by $\min \{h, 1\} \in L^{1}\left(\mathbb{R}^{n}\right) \cap L^{\infty}\left(\mathbb{R}^{n}\right)$, as required by (A2M).

Corollary 3.7. If $\varphi \in \Phi\left(\mathbb{R}^{n}\right)$ satisfies (A0)-(A2) and there exists $\gamma>1$ such that $s \mapsto s^{-\gamma} \varphi(x, s)$ is increasing for every $x \in \mathbb{R}^{n}$, then $M: L^{\varphi(\cdot)}\left(\mathbb{R}^{n}\right) \rightarrow L^{\varphi(\cdot)}\left(\mathbb{R}^{n}\right)$ is bounded.

Proof. As in subsection (A0), we find $\varphi_{1} \in \Phi_{1}$ with $\varphi_{1} \simeq \varphi$. Then $\varphi_{1}$ satisfies (A0M). By Lemma 3.1, $\varphi_{1}$ satisfies (A1) and (A2). A short calculation gives that $s \mapsto s^{-\gamma} \varphi_{1}(x, s)$ is increasing. By Corollary 3.4 and Lemma 3.6, (A1M) and (A2M) hold. Therefore by Theorem 2.7 the maximal operator is bounded on $L^{\varphi_{1}(\cdot)}\left(\mathbb{R}^{n}\right)$ and thus also on $L^{\varphi(\cdot)}\left(\mathbb{R}^{n}\right)$.

$\Phi$-functions are not totally well-behaved with respect to taking limits. Consider for instance $t^{p}$. As $p \rightarrow \infty$, the point-wise limit is $\infty \chi_{(1, \infty)}+\chi_{\{1\}}$, which is not leftcontinuous. For the equivalent $\Phi$-function $\frac{1}{p} t^{p}$ we have limit $\infty \chi_{(1, \infty)}$, which is what we want. Therefore, we need to chose the equivalent $\Phi$-function suitably to get a good limit.

We are especially interested in the behavior of $\varphi_{\infty}$ when $t \leqslant 1$. To this end we define

$$
\varphi_{2}(x, t):=\max \left\{\varphi_{1}(x, t), 2 t-1\right\} .
$$

Clearly $\varphi_{1} \leqslant \varphi_{2}$. For $t \leqslant \frac{1}{2}, \varphi_{2}=\varphi_{1}$. Since $\varphi \in \Phi_{1}\left(\mathbb{R}^{n}\right)$, we have $\varphi_{1}(x, 1)=1$ and $\varphi_{1}(x, t) \geqslant t$ for $t \geqslant 1$ by convexity. Thus $\varphi_{2}(x, t) \leqslant \varphi_{2}(x, 1)=1 \leqslant \varphi_{1}(x, 2 t)$ for $t \in\left[\frac{1}{2}, 1\right]$ and $\varphi_{2}(x, t) \leqslant 2 \varphi_{1}(x, t) \leqslant \varphi_{1}(x, 2 t)$ for $t>1$. In sum, we obtain $\varphi_{2} \simeq \varphi_{1} \simeq \varphi$ with $\varphi_{2}(x, 1) \equiv 1 \equiv \varphi_{2}^{-1}(x, 1)$.

Note that the right-derivative satisfies $\varphi_{2}^{\prime}\left(x, 1^{-}\right) \in[1,2]$ : here the lower bound follows from convexity by $\varphi_{2}^{\prime}(x, 1) \geqslant \varphi_{2}(x, 1)=1$ and the upper bound holds since if $\varphi_{2}^{\prime}\left(x, 1^{-}\right)>2$, then $\varphi_{2}(x, t)<2 t-1$ for some $t<1$ contrary to the construction of $\varphi_{2}$.

We consider then the limit $\left(\varphi_{2}\right)_{\infty}(t)=\lim \sup _{|x| \rightarrow \infty} \varphi_{2}(x, t)$. Clearly $\left(\varphi_{2}\right)_{\infty}(0)=0$ and $\left(\varphi_{2}\right)_{\infty}(1)=1$. For $t \in(0,1), 1=\left(\varphi_{2}\right)_{\infty}(1) \geqslant\left(\varphi_{2}\right)_{\infty}(t) \geqslant 2 t-1$ and hence $\left(\varphi_{2}\right)_{\infty}$ is left-continuous at 1 . By convexity of $\varphi_{2}, \varphi_{2}(x, t) \leqslant t \varphi_{2}(x, 1)=t$ on $[0,1]$ and hence $\left(\varphi_{2}\right)_{\infty}\left(0^{+}\right)=0$. Since $\left(\varphi_{2}\right)_{\infty}(t) \geqslant t$ for $t \geqslant 1$, we have $\lim _{t \rightarrow \infty}\left(\varphi_{2}\right)_{\infty}(t)=$ $\infty$.

To show that $\left(\varphi_{2}\right)_{\infty}$ is convex let $0 \leqslant t_{1}<t_{2}$ and $\theta \in(0,1)$. Choose $x_{i} \rightarrow \infty$ such that

$$
\left(\varphi_{2}\right)_{\infty}\left(\theta t_{1}+(1-\theta) t_{2}\right)=\lim _{i} \varphi_{2}\left(x_{i}, \theta t_{1}+(1-\theta) t_{2}\right) .
$$

By convexity of $\varphi_{2}$,

$$
\begin{aligned}
\lim _{i} \varphi_{2}\left(x_{i}, \theta t_{1}+(1-\theta) t_{2}\right) & \leqslant \lim _{i}\left[\theta \varphi_{2}\left(x_{i}, t_{1}\right)+(1-\theta) \varphi_{2}\left(x_{i}, t_{2}\right)\right] \\
& \leqslant \theta\left(\varphi_{2}\right)_{\infty}\left(t_{1}\right)+(1-\theta)\left(\varphi_{2}\right)_{\infty}\left(t_{2}\right),
\end{aligned}
$$

so $\left(\varphi_{2}\right)_{\infty}$ is convex, as well.

Since $\left(\varphi_{2}\right)_{\infty}$ is convex and increasing on $[0,1]$, and left-continuous at 1 , it is actually continuous on $[0,1]$.

Remark 3.8. In the variable exponent setting, (A2) is equivalent to Nekvinda's decay condition (see [12, Remark 4.2.8] for details), which is a weaker version of the logHölder decay condition. 
Remark 3.9. Note that (A2) implies also the equivalence of norms: indeed, this is a general property of solid Banach spaces, as the following well-known argument shows. (Recall that a space is solid if $|f| \leqslant|g|$ implies $\|f\| \leqslant\|g\|$.) If $\|f\|_{A} \not\|f\|_{B}$, then we can choose $f_{i}$ such that $\left\|f_{i}\right\|_{A} \geqslant 3^{i}$ but $\left\|f_{i}\right\|_{B} \leqslant 1$. Now for $g:=\sum_{i} 2^{-i}\left|f_{i}\right|$ we have

$$
\|g\|_{A} \geqslant\left\|2^{-i} f_{i}\right\|_{A} \geqslant(3 / 2)^{i} \rightarrow \infty
$$

and $\|g\|_{B} \leqslant \sum_{i} 2^{-i}=1$ so that $g \in B \backslash A$. Hence $A \neq B$. The implication $A=B \Rightarrow\|\cdot\|_{A} \approx\|\cdot\|_{B}$ follows by contraposition.

\section{THE FINAL NORMALIZATION}

Next we make the final normalization of $\varphi$ satisfying (A0)-(A2) by setting

$$
\bar{\varphi}(x, t)= \begin{cases}2 \varphi_{2}(x, t)-1, & \text { if } t \geqslant 1 \\ \left(\varphi_{2}\right)_{\infty}(t), & \text { if } t<1 .\end{cases}
$$

Lemma 4.1. If $\varphi \in \Phi\left(\mathbb{R}^{n}\right)$ satisfies (AO)-(A2), then $\bar{\varphi} \in \Phi_{1}\left(\mathbb{R}^{n}\right)$.

Proof. For the convexity we have to show that the $\bar{\varphi}^{\prime}(x, \cdot)$ is increasing for every $x \in$ $\mathbb{R}^{n}$. We have

$$
\bar{\varphi}^{\prime}(x, t)= \begin{cases}2 \varphi_{2}^{\prime}(x, t), & \text { if } t \geqslant 1 \\ \left(\varphi_{2}\right)_{\infty}^{\prime}(t), & \text { if } t<1 .\end{cases}
$$

By convexity each of the parts is increasing. At $1,2 \varphi_{2}^{\prime}(x, 1) \geqslant 2$ and $\lim _{t \rightarrow 1^{-}}\left(\varphi_{2}\right)_{\infty}^{\prime}(t) \leqslant$ 2 (see discussion regarding (A2)), so the right-derivative is increasing also there.

The function $\bar{\varphi}$ is continuous since both $\varphi_{2}$ and $\left(\varphi_{2}\right)_{\infty}$ are continuous and $\varphi_{2}(x, 1)=$ $\left(\varphi_{2}\right)_{\infty}\left(1^{-}\right)=1$. Thus we have that $\bar{\varphi}^{-1}(x, 1) \leqslant 1$. In the discussion on (A2), we noted that $\varphi_{2}(x, t) \leqslant t$ on $[0,1]$. These together give $\bar{\varphi}^{-1}(x, 1) \equiv 1$.

The conditions $\bar{\varphi}(x, 0)=\bar{\varphi}\left(x, 0^{+}\right)=0$ and $\lim _{t \rightarrow \infty} \bar{\varphi}(t)=\infty$ follow from the same conditions for $\varphi_{2}$ and $\left(\varphi_{2}\right)_{\infty}$.

Proposition 4.2. If $\varphi \in \Phi\left(\mathbb{R}^{n}\right)$ satisfies $(A O)-(A 2)$, then $L^{\varphi(\cdot)}=L^{\bar{\varphi}(\cdot)}$ with equivalent norms.

Proof. Since $\varphi \simeq \varphi_{2}$, it suffices to show that $L^{\varphi_{2}(\cdot)}=L^{\bar{\varphi}(\cdot)}$.

Let $g \in L^{\varphi_{2}(\cdot)}\left(\mathbb{R}^{n}\right)$ and set $f:=g /\|g\|_{\varphi_{2}(\cdot)}$. We divide $f$ into two parts, $f_{1}=$ $f \chi_{\{|f|<1\}}$ and $f_{2}=f \chi_{\{|f| \geqslant 1\}}$. By (A2), and since $\|f\|_{\varphi_{2}(\cdot)}=1$,

$$
\begin{aligned}
\left\|f_{1}\right\|_{\bar{\varphi}(\cdot)} & =\left\|f_{1}\right\|_{\left(\varphi_{2}\right)_{\infty}} \leqslant\left\|f_{1}\right\|_{L^{\left(\varphi_{2}\right) \infty \cap L^{\infty}}} \\
& \approx\left\|f_{1}\right\|_{L^{\varphi_{2}(\cdot)} \cap L^{\infty}}=\max \left\{\left\|f_{1}\right\|_{\varphi_{2}(\cdot)},\left\|f_{1}\right\|_{\infty}\right\} \leqslant 1 .
\end{aligned}
$$

If $\left|f_{2}(x)\right| \geqslant 1$, then

$$
\bar{\varphi}\left(x,\left|f_{2}\right|\right)=2 \varphi_{2}\left(x,\left|f_{2}\right|\right)-1 \leqslant 2 \varphi_{2}\left(x,\left|f_{2}\right|\right) .
$$

Otherwise, $\left|f_{2}(x)\right|=0$, and the inequality holds as well. Thus $\left\|f_{2}\right\|_{\bar{\varphi}(\cdot)} \lesssim\left\|f_{2}\right\|_{\varphi_{2}(\cdot)} \leqslant$ 1 and hence

$$
\|g /\| g\left\|_{\varphi_{2}(\cdot)}\right\|_{\bar{\varphi}(\cdot)}=\|f\|_{\bar{\varphi}(\cdot)} \leqslant\left\|f_{1}\right\|_{\bar{\varphi}(\cdot)}+\left\|f_{2}\right\|_{\bar{\varphi}(\cdot)} \lesssim 1
$$

so that $\|g\|_{\bar{\varphi}(\cdot)} \lesssim\|g\|_{\varphi_{2}(\cdot)}$. The opposite inequality is proved similarly.

Remark 4.3. While the spaces in the previous proposition are the same, it is not necessary that $\varphi \simeq \bar{\varphi}$. For instance, if $\varphi(x, t):=\max \left\{t-\frac{1}{2+|x|}, 0\right\}$ then $\varphi\left(0, \frac{1}{2}\right)=0$ yet $\varphi_{\infty}(t)=t>0$ for all $t>0$. Then for every $\beta \in(0,2), \bar{\varphi}\left(0, \beta \frac{1}{2}\right)=\beta \frac{1}{2} \nless 0=\varphi\left(0, \frac{1}{2}\right)$, so the $\Phi$-functions are not equivalent. 
Proposition 4.2 and Corollary 3.7 now imply the following:

Corollary 4.4. If $\varphi \in \Phi\left(\mathbb{R}^{n}\right)$ satisfies (AO)-(A2) and there exists $\gamma>1$ such that $s \mapsto s^{-\gamma} \varphi(x, s)$ is increasing for every $x \in \mathbb{R}^{n}$, then $M: L^{\bar{\varphi}(\cdot)}\left(\mathbb{R}^{n}\right) \rightarrow L^{\bar{\varphi}(\cdot)}\left(\mathbb{R}^{n}\right)$ is bounded.

Note the range of permissible values of $t$ in the following proposition, including also $[0,1]$. This is sometimes very useful, e.g. in Proposition 4.8.

Proposition 4.5. If $\varphi \in \Phi\left(\mathbb{R}^{n}\right)$ satisfies (AO)-(A2), then there exists $\beta \in(0,1)$ such that

$$
\beta \bar{\varphi}^{-1}(x, t) \leqslant \bar{\varphi}^{-1}(y, t)
$$

for every $t \in\left[0, \frac{1}{|B|}\right]$, every $x, y \in B$ and every ball $B$.

Proof. If $t \leqslant 1$, then $\bar{\varphi}$ is independent of $x$, so the claim is trivial. Thus it remains only to consider the case $t>1$. Then by Lemma 3.3 the inequality holds if and only if

$$
\bar{\varphi}(x, \beta s) \leqslant \bar{\varphi}(y, s)
$$

for every $s \in\left[1, \bar{\varphi}^{-1}\left(y, \frac{1}{|B|}\right)\right]$, Since $\bar{\varphi} \geqslant \varphi_{2}$, such $s$ satisfies $s \in\left[1, \varphi_{2}^{-1}\left(y, \frac{1}{|B|}\right)\right]$. If $\beta s \geqslant 1$, then using Lemma 3.3 for $\varphi_{2}$ we calculate

$$
\bar{\varphi}(x, \beta s)=2 \varphi_{2}(x, \beta s)-1 \leqslant 2 \varphi_{2}(y, s)-1=\bar{\varphi}(y, s) .
$$

If $\beta s<1$, then $\bar{\varphi}(x, \beta s) \leqslant \bar{\varphi}(x, 1)=1 \leqslant \bar{\varphi}(y, 1) \leqslant \bar{\varphi}(y, s)$, so the inequality holds in both cases.

In view of the previous proposition and the observations of Section 3, we make the following definition. Note that a normalized $\Phi$-function satisfies assumptions (A0)(A2).

Definition 4.7. We say that $\varphi \in \Phi\left(\mathbb{R}^{n}\right)$ is a normalized $\Phi$-function if $\varphi(x, t)=\varphi_{\infty}(t)$ for $t \in[0,1], \varphi_{\infty}(1) \in(0, \infty)$, and there exists $\beta>0$ such that

$$
\beta \varphi^{-1}(x, t) \leqslant \varphi^{-1}(y, t)
$$

for every $t \in\left[0, \frac{1}{|B|}\right]$, every $x, y \in B$ and every ball $B$.

Proposition 4.5 says that instead of studying $\varphi \in \Phi\left(\mathbb{R}^{n}\right)$ which satisfies (A0)-(A2) we can study the normalized $\Phi$-function $\bar{\varphi}$. This sometimes leads to great simplifications in proofs, as the following result shows (compare this to [12, Section 4.5], and see Remark 4.9).

Proposition 4.8. Suppose that $\varphi \in \Phi\left(\mathbb{R}^{n}\right)$ is normalized. Let $B \ni x$ be a ball. Then

$$
\left\|\chi_{B}\right\|_{\varphi(\cdot)} \leqslant \frac{1}{\beta \varphi^{-1}\left(x, \frac{1}{|B|}\right)} .
$$

Proof. By assumption

$$
\varphi\left(y, \beta \varphi^{-1}\left(x, \frac{1}{|B|}\right)\right) \leqslant \varphi\left(y, \varphi^{-1}\left(y, \frac{1}{|B|}\right)\right) \leqslant \frac{1}{|B|}
$$

when $x, y \in B$, and hence

$$
\varrho_{\varphi(\cdot)}\left(\beta \varphi^{-1}\left(x, \frac{1}{|B|}\right) \chi_{B}\right)=\int_{B} \varphi\left(y, \beta \varphi^{-1}\left(x, \frac{1}{|B|}\right)\right) d y \leqslant 1 .
$$


Remark 4.9. In some regards it is actually easier to study general normalized $\Phi$ functions than the special case of variable exponent spaces: the normalization allows us to omit the error term which commonly appears in the variable exponent case. This is a consequence of the fact that $\varphi(x, t)=\varphi_{\infty}(t)$ in the normalized case for small $t$, whereas only $t^{p(x)} \leqslant t^{p \infty}+h(x)$ holds in the variable exponent case; the function $h$ leads to the error term.

\section{DuALity}

The conjugate $\Phi$-function of $\varphi$ is defined by

$$
\varphi^{*}(t):=\sup _{s>0}(s t-\varphi(s)) .
$$

Note that $\varphi^{* *}=\varphi\left[12\right.$, Corollary 2.6.3]. For $\gamma>1$, the Hölder conjugate $\gamma^{\prime}$ is defined by $\frac{1}{\gamma}+\frac{1}{\gamma^{\prime}}=1$. If $\varphi(t)=\frac{1}{p} t^{p}$, then $\varphi^{*}(t)=\frac{1}{p^{\prime}} t^{p^{\prime}}$ and we get the usual Lebesgue duality. The dual is defined for generalized $\Phi$-functions point-wise. Note that conjugating preserves equivalence, i.e. if $\varphi \simeq \psi$, then $\varphi^{*} \simeq \psi^{*}$ [12, Lemma 2.6.4].

Differentiating $s t-\varphi(s)$ to find the maximum, we obtain that $\varphi^{*}(t)=t\left(\varphi^{\prime}\right)^{-1}(t)-$ $\varphi\left(\left(\varphi^{\prime}\right)^{-1}(t)\right)$, where $\left(\varphi^{\prime}\right)^{-1}(t)$ is the right-continuous inverse:

$$
\left(\varphi^{\prime}\right)^{-1}(t):=\sup \left\{\tau \geqslant 0 \mid \varphi^{\prime}(\tau) \leqslant t\right\} .
$$

For duality arguments we often need functions nicer than $\Phi$-functions: $N$-functions are those (continuous) $\Phi$-functions which satisfy $\varphi(t) \in(0, \infty)$ when $t>0, \lim _{t \rightarrow 0^{+}} \frac{\varphi(t)}{t}=$ 0 and $\lim _{t \rightarrow \infty} \frac{\varphi(t)}{t}=\infty$. The set of $N$-functions is denoted by $N$. Note that $N$ functions are strictly increasing. For example, if $\varphi(t)=t^{p}$, then $\varphi \in N$ if and only if $p \in(1, \infty)$.

We say that $\varphi \in \Phi\left(\mathbb{R}^{n}\right)$ is a (generalized) uniform $N$-function if there exists $\eta, \xi \in$ $N$ such that

$$
\eta(t) \leqslant \varphi(x, t) \leqslant \xi(t)
$$

for every $x \in \mathbb{R}^{n}$ and $t \geqslant 0$. The set of uniform $N$-functions is denoted by $N\left(\mathbb{R}^{n}\right)$. We set $N_{1}\left(\mathbb{R}^{n}\right):=\Phi_{1}\left(\mathbb{R}^{n}\right) \cap N\left(\mathbb{R}^{n}\right)$.

In the variable exponent case, $t^{p(\cdot)}$ is uniformly $N$ if and only of inf $p>1$ and $\sup p<\infty$, whereas the non-uniform case requires only $1<p<\infty$ point-wise. The latter condition has turned out to be nearly useless in $L^{p(\cdot)}$-research, so it is natural to consider here only the uniform case.

Proposition 5.1. If $\varphi \in N\left(\mathbb{R}^{n}\right)$ satisfies (AO)-(A2), then $\bar{\varphi} \in N_{1}\left(\mathbb{R}^{n}\right)$.

Proof. By Lemma 4.1, $\bar{\varphi} \in \Phi_{1}\left(\mathbb{R}^{n}\right)$. We need to check that the normalizations do not destroy the functions $\eta$ and $\xi$. By (A0), there exists $\beta \in(0,1)$ such that $\beta \leqslant$ $\varphi^{-1}(x, 1) \leqslant 1 / \beta$. First we set $\eta_{1}(t):=\eta(\beta t)$ and $\xi_{1}(t):=\xi(t / \beta)$. Then $\eta_{1} \leqslant \varphi_{1} \leqslant \xi_{1}$. As before, $\eta_{2}(t)=\max \left\{\eta_{1}(t), 2 t-1\right\}$, similarly for $\xi$. Then also $\eta_{2} \leqslant \varphi_{2} \leqslant \xi_{2}$, and we easily see that $\eta_{2}$ and $\xi_{2}$ are still $N$-functions. Furthermore, $\bar{\eta}=\max \left\{\eta_{2}, 2 \eta_{2}-1\right\}$ is an $N$-function minorizing $\bar{\varphi}$, similarly for $\bar{\xi}$.

Lemma 5.2. Let $\varphi \in N$ and $\gamma>1$. Then $s \mapsto s^{-\gamma} \varphi(s)$ is increasing if and only if $s \mapsto s^{-\gamma^{\prime}} \varphi^{*}(s)$ is decreasing.

Proof. We note that $t \mapsto t^{-\gamma} \varphi(t)$ is increasing if and only if $D\left(t^{-\gamma} \varphi(t)\right) \geqslant 0$, i.e. $t \varphi^{\prime}(t) \geqslant \gamma \varphi(t)$. Since $\varphi$ is continuous, we conclude from this that

$$
t \varphi^{\prime}\left(t^{-}\right) \geqslant \gamma \varphi\left(t^{-}\right)=\gamma \varphi(t) .
$$


On the other hand, as noted after the definition of $\varphi^{*}$, with $t:=\left(\varphi^{\prime}\right)^{-1}(s)$,

$$
\varphi^{*}(s)=s t-\varphi(t) \geqslant s t-\frac{1}{\gamma} t \varphi^{\prime}\left(t^{-}\right) \text {. }
$$

By [12, Remark 2.6.9], $t=\left(\varphi^{*}\right)^{\prime}(s)$ and by [12, (2.6.14)], $\varphi^{\prime}\left(\left(\varphi^{*}\right)^{\prime}(s)-\varepsilon\right) \leqslant s$ for all $\varepsilon>0$, so that $\varphi^{\prime}\left(t^{-}\right) \leqslant s$. In the previous inequality, this gives $\varphi^{*}(s) \geqslant \frac{1}{\gamma^{\prime}} s t=$ $\frac{1}{\gamma^{\prime}} s\left(\varphi^{*}\right)^{\prime}(s)$, which is equivalent to $D\left(s^{-\gamma^{\prime}} \varphi^{*}(s)\right) \leqslant 0$, as was to be shown.

Proposition 5.3. If $\varphi \in N_{1}\left(\mathbb{R}^{n}\right)$ is normalized, then also $\varphi^{*} \in N\left(\mathbb{R}^{n}\right)$ is normalized.

Proof. First we note that $\eta^{*}, \xi^{*} \in N$ by [12, Theorem 2.6.8]. The inequalities $\eta(t) \leqslant$ $\varphi(x, t) \leqslant \xi(t)$ yield that $\xi^{*}(t) \leqslant \varphi^{*}(x, t) \leqslant \eta^{*}(t)$ by [12, Lemma 2.6.4], and thus $\varphi^{*} \in N\left(\mathbb{R}^{n}\right)$.

By [12, Lemma 2.6.11], $t \leqslant \psi^{-1}(t)\left(\psi^{*}\right)^{-1}(t) \leqslant 2 t$ for $\psi \in N$. Let $x, y \in B$ and $t \leqslant \frac{1}{|B|}$. Then

$$
\frac{\beta}{2}\left(\varphi^{*}\right)^{-1}(x, t) \leqslant \frac{\beta t}{\varphi^{-1}(x, t)} \leqslant \frac{t}{\varphi^{-1}(y, t)} \leqslant\left(\varphi^{*}\right)^{-1}(y, t) .
$$

Furthermore, $\varphi(x, s) \geqslant \varphi(x, 1) s=s$ when $s \geqslant 1$ (since $\varphi$ is convex). When $t \leqslant 1$ and $s \geqslant 1$, it follows that $s t-\varphi(x, s) \leqslant s(t-1) \leqslant 0$. Hence, for $t \leqslant 1$,

$$
\varphi^{*}(x, t)=\sup _{s>0}(s t-\varphi(x, s))=\sup _{s \in[0,1]}(s t-\varphi(x, s))=\sup _{s \in[0,1]}\left(s t-\varphi_{\infty}(s)\right)=\varphi_{\infty}^{*}(t)
$$

is independent of $x$. Since $0=\lim _{t \rightarrow 0^{+}} \frac{\varphi(x, t)}{t}=\lim _{t \rightarrow 0^{+}} \frac{\varphi_{\infty}(t)}{t}$ we obtain that $1 \geqslant$ $\varphi_{\infty}^{*}(1)=\sup _{s \in[0,1]}\left(s-\varphi_{\infty}(s)\right)>0$. Therefore we have shown that it is a normalized $N$-function.

\section{The Riesz Potential AND THE SOBOLEV EMBEDDing}

Let $0<\alpha<n$. For measurable $f$ we define $I_{\alpha} f: \mathbb{R}^{n} \rightarrow[0, \infty]$ by

$$
I_{\alpha} f(x):=\int_{\mathbb{R}^{n}} \frac{|f(y)|}{|x-y|^{n-\alpha}} d y .
$$

The operator $I_{\alpha}$ is called the Riesz potential operator.

Lemma 6.1. For $\varphi \in \Phi\left(\mathbb{R}^{n}\right)$ we write $\hat{\varphi}(x, t):=\varphi^{*}\left(x, t^{\frac{n-\alpha}{n}}\right)$. Assume that $M$ is bounded from $L^{\hat{\varphi}(\cdot)}\left(\mathbb{R}^{n}\right)$ to itself. Let $x \in \mathbb{R}^{n}, \delta>0$, and $f \in L^{\varphi(\cdot)}\left(\mathbb{R}^{n}\right)$ with $\|f\|_{\varphi(\cdot)} \leqslant$ 1. Then

$$
\int_{\mathbb{R}^{n} \backslash B(x, \delta)} \frac{|f(y)|}{|x-y|^{n-\alpha}} d y \lesssim|B(x, \delta)|^{\frac{\alpha-n}{n}}\left\|\chi_{B(x, \delta)}\right\|_{\varphi^{*}(\cdot)} .
$$

Proof. Set $B:=B(x, \delta)$. We start with Hölder's inequality and take into account that $\|f\|_{\varphi(\cdot)} \leqslant 1$ :

$$
\begin{aligned}
\int_{\mathbb{R}^{n} \backslash B} \frac{|f(y)|}{|x-y|^{n-\alpha}} d y & \leqslant 2\|f\|_{\varphi(\cdot)}\left\|\chi_{\mathbb{R}^{n} \backslash B}|x-\cdot|^{\alpha-n}\right\|_{\varphi^{*}(\cdot)} \\
& \leqslant 2\left\|\chi_{\mathbb{R}^{n} \backslash B}|x-\cdot|^{-n}\right\|_{\hat{\varphi}(\cdot)}^{\frac{n-\alpha}{n}} .
\end{aligned}
$$

Next we note that, for all $y \in \mathbb{R}^{n} \backslash B$,

$$
M\left(\chi_{B}|B|^{-1}\right)(y) \geqslant f_{B(y, 2|x-y|)} \chi_{B}(z)|B|^{-1} d z=|B(y, 2|x-y|)|^{-1}=c|x-y|^{-n} \text {. }
$$


Therefore $\chi_{\mathbb{R}^{n} \backslash B}(y)|x-y|^{-n} \lesssim M\left(\chi_{B}|B|^{-1}\right)(y)$ for all $y \in \mathbb{R}^{n}$. Combining the previous estimates and using the boundedness of $M$, we find that

$$
\begin{aligned}
\int_{\mathbb{R}^{n} \backslash B} \frac{|f(y)|}{|x-y|^{n-\alpha}} d y & \lesssim\left\|M\left(\chi_{B}|B|^{-1}\right)\right\|_{\hat{\varphi}(\cdot)}^{\frac{n-\alpha}{n}}=|B|^{\frac{\alpha-n}{n}}\left\|M\left(\chi_{B}\right)\right\|_{\hat{\varphi}(\cdot)}^{\frac{n-\alpha}{n}} \\
& \lesssim|B|^{\frac{\alpha-n}{n}}\left\|\chi_{B}\right\|_{\hat{\varphi}(\cdot)}^{\frac{n-\alpha}{n}}=|B|^{\frac{\alpha-n}{n}}\left\|\chi_{B}\right\|_{\varphi^{*}(\cdot)} .
\end{aligned}
$$

Recall that a function is almost decreasing if $f(x) \leqslant Q f(y)$ when $x>y$, for some fixed $Q \in[1, \infty)$. Almost increasing is defined analogously.

Lemma 6.2. Let $\varphi \in N_{1}\left(\mathbb{R}^{n}\right)$ be normalized and suppose that $s \mapsto s^{\frac{\varepsilon-n}{\alpha}} \varphi(x, s)$ is almost decreasing for every $x \in \mathbb{R}^{n}$. Then

$$
I_{\alpha} f(x) \lesssim \varphi(x, M f(x))^{-\frac{\alpha}{n}} M f(x) \quad \text { a.e. }
$$

for every $f \in L^{\varphi(\cdot)}\left(\mathbb{R}^{n}\right)$ with $\|f\|_{\varphi(\cdot)} \leqslant 1$.

Proof. Let us write $B:=B(x, \delta)$. We divide the Riesz-potential into two parts:

$$
I_{\alpha} f(x)=\int_{B} \frac{|f(y)|}{|x-y|^{n-\alpha}} d y+\int_{\mathbb{R}^{n} \backslash B} \frac{|f(y)|}{|x-y|^{n-\alpha}} d y .
$$

In the first part we split the integration domain into annuli and use the definition of $M$ :

$$
\begin{aligned}
\int_{B} \frac{|f(y)|}{|x-y|^{n-\alpha}} d y & \leqslant \sum_{k=1}^{\infty}\left(\delta 2^{-k}\right)^{\alpha-n} \int_{2^{-k} \delta \leqslant|x-y|<2^{-k+1} \delta}|f(y)| d y \\
& \lesssim \sum_{k=1}^{\infty}\left(\delta 2^{-k}\right)^{\alpha} f_{|x-y|<2^{-k+1} \delta}|f(y)| d y \\
& \leqslant \delta^{\alpha} \sum_{k \in \mathbb{N}} 2^{-\alpha k} M f(x) \\
& =c|B|^{\frac{\alpha}{n}} M f(x) .
\end{aligned}
$$

Let $\hat{\varphi}(x, t)=\varphi^{*}\left(x, t^{\frac{n-\alpha}{n}}\right)$ be as is Lemma 6.1. By Proposition 5.3, $\varphi^{*}$ is normalized. Thus $\varphi^{*}$ satisfies (A0)-(A2). Further $\hat{\varphi}^{-1}(x, t)=\left(\left(\varphi^{*}\right)^{-1}(x, t)\right)^{\frac{n}{n-\alpha}}$, and so $\hat{\varphi}$ inherits (A0)-(A2) from $\varphi^{*}$.

Set $\gamma:=\frac{n-\varepsilon}{\alpha}$ and define $\psi(x, t):=t^{\gamma} \sup _{s \geqslant t} s^{-\gamma} \varphi(x, s)$ for $t \geqslant 0$. This definition directly implies that $t^{-\gamma} \psi(x, t)$ is decreasing and $\psi \geqslant \varphi$. Since $s^{-\gamma} \varphi(x, s)$ is almost decreasing by assumption, $\psi \leqslant Q \varphi$, so that $\varphi \simeq \psi$. By Lemma 5.2, $t^{-\gamma^{\prime}} \psi^{*}(x, t)$ is increasing, and since $\varphi^{*} \simeq \psi^{*}$ it follows that $t^{-\gamma^{\prime}} \varphi^{*}(x, t)$ is almost increasing. Therefore, with $s=t^{\frac{n-\alpha}{n}}$,

$$
t^{-\gamma^{\prime} \frac{n-\alpha}{n}} \hat{\varphi}(x, t)=t^{-\gamma^{\prime} \frac{n-\alpha}{n}} \varphi^{*}\left(x, t^{\frac{n-\alpha}{n}}\right)=s^{-\gamma^{\prime}} \varphi^{*}(x, s)
$$

is almost increasing. A calculation yields that $\hat{\gamma}:=\gamma^{\prime} \frac{n-\alpha}{n}>1$. Therefore $\hat{\varphi}$ is equivalent to a $\Phi$-function $\xi$ with $t^{-\hat{\gamma}} \xi(x, t)$ increasing (cf. [19, Section 5]). Since $\hat{\varphi} \simeq \xi$, also (A0)-(A2) hold. By Corollary 3.7, $M$ is bounded on $L^{\xi(\cdot)}$, and hence also on $L^{\hat{\varphi}(\cdot)}$.

Therefore, the assumptions of Lemma 6.1 hold, and it follows that

$$
\int_{\mathbb{R}^{n} \backslash B} \frac{|f(y)|}{|x-y|^{n-\alpha}} d y \lesssim|B|^{\frac{\alpha-n}{n}}\left\|\chi_{B}\right\|_{\varphi^{*}(\cdot)}
$$

provided $\|f\|_{\varphi(\cdot)} \leqslant 1$. 
We combine (6.3) with Propositions 4.8 and 5.3, and obtain that

$$
I_{\alpha} f(x) \lesssim|B|^{\frac{\alpha}{n}} M f(x)+|B|^{\frac{\alpha-n}{n}}\left\|\chi_{B}\right\|_{\varphi^{*}(\cdot)} \leqslant|B|^{\frac{\alpha}{n}} M f(x)+\frac{|B|^{\frac{\alpha-n}{n}}}{\beta\left(\varphi^{*}\right)^{-1}\left(x, \frac{1}{|B|}\right)} .
$$

Now $\left(\varphi^{*}\right)^{-1}(x, t) \approx t / \varphi^{-1}(x, t)$ by [12, Lemma 2.6.11] and so

$$
I_{\alpha} f(x) \lesssim|B|^{\frac{\alpha}{n}} M f(x)+|B|^{\frac{\alpha}{n}} \varphi^{-1}\left(x, \frac{1}{|B|}\right) .
$$

When $M f(x)<\infty$, we choose the radius $\delta$ such that $M f(x)=\varphi^{-1}\left(x, \frac{1}{|B|}\right)$, i.e. $|B|=1 / \varphi(x, M f(x))$. Thus

$$
I_{\alpha} f(x) \lesssim \varphi(x, M f(x))^{-\frac{\alpha}{n}} M f(x) \quad \text { a.e. }
$$

Lemma 6.4. Let $\alpha>0, \varphi \in N\left(\mathbb{R}^{n}\right)$ with $t \mapsto t^{-\frac{n}{\alpha}} \varphi(x, t)$ strictly decreasing to 0 for every $x \in \mathbb{R}^{n}$ and let $\lambda(x, t):=t \varphi(x, t)^{-\frac{\alpha}{n}}$. Then $\varphi \circ\left(\lambda^{-1}\right)$ is equivalent to a convex $\Phi$-function.

By $\varphi \circ\left(\lambda^{-1}\right)$ we mean the function $(x, t) \mapsto \varphi\left(x, \lambda^{-1}(x, t)\right)$.

Proof. Since the claim is point-wise in nature, we drop the variable $x$ for the rest of the proof.

Let us denote $\psi:=\varphi \circ\left(\lambda^{-1}\right)$. Since $t^{-\frac{n}{\alpha}} \varphi(t) \rightarrow 0$ we find that $\lambda(t) \rightarrow \infty$ as $t \rightarrow \infty$. Thus also $\psi(t) \rightarrow \infty$ as $t \rightarrow \infty$. The function

$$
\lambda(s)^{\frac{n}{\alpha}}=\frac{s^{n / \alpha}}{\varphi(s)}
$$

is strictly increasing by assumption, so the same holds for $\lambda^{-1}$. Furthermore, with $s=\lambda(t)$, the fraction

$$
\frac{\lambda^{-1}(s)}{s}=\frac{t}{\lambda(t)}=\varphi(t)^{\frac{\alpha}{n}}
$$

is increasing in $t$ (since $\varphi$ is increasing), hence in $s$ as well. Since $t \mapsto \varphi(t) / t$ is increasing (due to convexity of $\varphi$ and $\varphi(0)=0$ ) this yields that

$$
\frac{\psi(t)}{t}=\frac{\varphi\left(\lambda^{-1}(t)\right)}{\lambda^{-1}(t)} \frac{\lambda^{-1}(t)}{t}
$$

is also increasing. Since $\frac{\psi(t)}{t}$ is increasing, we obtain $\lim _{t \rightarrow 0^{+}} \psi(t)=0$. Thus it follows from Lemma 2.6 that $\psi$ is equivalent to a convex function.

The previous lemma shows that the next definition makes sense.

Definition 6.5. Let $\alpha>0$ and $\varphi \in N\left(\mathbb{R}^{n}\right)$ with $t \mapsto t^{-\frac{n}{\alpha}} \varphi(x, t)$ strictly decreasing to 0 for every fixed $x$. We define $\lambda(x, t):=t \varphi(x, t)^{-\frac{\alpha}{n}}$ and let $\varphi_{\alpha}^{\#} \in \Phi\left(\mathbb{R}^{n}\right)$ be a $\Phi$-function equivalent to $\varphi \circ\left(\lambda^{-1}\right)$ (which exists by Lemma 6.4).

Lemma 6.6. If $\varphi \in \Phi\left(\mathbb{R}^{n}\right)$ satisfies assumptions (A0)-(A2) and $t \mapsto t^{\gamma} \varphi(x, t), \gamma<0$, is decreasing, then $t \mapsto t^{\gamma} \bar{\varphi}(x, t)$ is almost decreasing.

Proof. We prove first that $t \mapsto t^{\gamma} \varphi_{2}(x, t)$ is almost decreasing. Since $\varphi \simeq \varphi_{2}$, for $s<t$,

$$
\begin{aligned}
s^{\gamma} \varphi_{2}(x, s) & \geqslant s^{\gamma} \varphi(x, s / L)=L^{\gamma}(s / L)^{\gamma} \varphi(x, s / L) \geqslant L^{\gamma}(t / L)^{\gamma} \varphi(x, t / L) \\
& \geqslant L^{\gamma}(L t)^{\gamma} \varphi(x, L t) \geqslant L^{\gamma}(L t)^{\gamma} \varphi_{2}(x, t)=L^{2 \gamma} t^{\gamma} \varphi_{2}(x, t) .
\end{aligned}
$$


Using this we obtain the same property for $\bar{\varphi}$ : If $0<s<t \leqslant 1$, then

$$
s^{\gamma} \bar{\varphi}(x, s)=\limsup _{|z| \rightarrow \infty} s^{\gamma} \varphi_{2}(z, s) \geqslant L^{2 \gamma} \limsup _{|z| \rightarrow \infty} t^{\gamma} \varphi_{2}(z, t)=L^{2 \gamma} t^{\gamma} \bar{\varphi}(x, t),
$$

and if $1 \leqslant s<t$, then

$$
\begin{aligned}
s^{\gamma} \bar{\varphi}(x, s) & =s^{\gamma}\left(2 \varphi_{2}(x, s)-1\right) \geqslant s^{\gamma} \varphi_{2}(x, s) \\
& \geqslant L^{2 \gamma} t^{\gamma} \varphi_{2}(x, t) \geqslant \frac{1}{2} L^{2 \gamma} t^{\gamma}\left(2 \varphi_{2}(x, t)-1\right)=\frac{1}{2} L^{2 \gamma} t^{\gamma} \bar{\varphi}(x, t) .
\end{aligned}
$$

Since the function is almost decreasing on $(0,1]$ and $[1, \infty)$, it is almost decreasing on the union as well.

Lemma 6.7. Let $\varphi \in N\left(\mathbb{R}^{n}\right)$ satisfy assumptions $(A 0)-(A 2)$ and let $t \mapsto t^{-\frac{n}{\alpha}} \varphi(x, t)$ be strictly decreasing to 0 . Then $L^{\varphi_{\alpha}^{\#}(\cdot)}\left(\mathbb{R}^{n}\right)=L^{\bar{\varphi}_{\alpha}^{\#}(\cdot)}\left(\mathbb{R}^{n}\right)$.

Proof. By Theorem 2.8.1 of [12], $L^{\psi(\cdot)}\left(\mathbb{R}^{n}\right) \subset L^{\varphi(\cdot)}\left(\mathbb{R}^{n}\right)$ if and only if there exist $\beta>0$ and $h \in L^{1}\left(\mathbb{R}^{n}\right)$ such that $\varphi(x, \beta t) \leqslant \psi(x, t)+h(x)$. This can be equivalently written as $\psi^{-1}(x, s) \lesssim \varphi^{-1}(x, s+h(x))$.

Let us show that $L^{\bar{\varphi}_{\alpha}^{\#}(\cdot)}\left(\mathbb{R}^{n}\right) \subset L^{\varphi_{\alpha}^{\#}(\cdot)}\left(\mathbb{R}^{n}\right)$; the reverse implication follows analogously. The inclusion is equivalent to the inequality

$$
\left(\bar{\varphi}_{\alpha}^{\#}\right)^{-1}(x, s) \lesssim\left(\varphi_{\alpha}^{\#}\right)^{-1}(x, s+h(x)) .
$$

By Lemma 6.6, $t \mapsto t^{-\frac{n}{\alpha}} \bar{\varphi}(x, t)$ is almost decreasing which is equivalent to $t \mapsto$ $t^{-\frac{\alpha}{n}} \bar{\varphi}^{-1}(x, t)$ being almost increasing. By the definition of $\bar{\varphi}_{\alpha}^{\#}$ and the almost increasing property, we obtain that

$$
\left(\bar{\varphi}_{\alpha}^{\#}\right)^{-1}(x, s) \approx \bar{\lambda}\left(\bar{\varphi}^{-1}(x, s)\right)=\frac{\bar{\varphi}^{-1}(x, s)}{\bar{\varphi}\left(x, \bar{\varphi}^{-1}(x, s)\right)^{\alpha / n}}=\frac{\bar{\varphi}^{-1}(x, s)}{s^{\alpha / n}} \lesssim \frac{\bar{\varphi}^{-1}(x, s+h(x))}{(s+h(x))^{\alpha / n}},
$$

where $\bar{\lambda}(x, t)=t \bar{\varphi}(x, t)^{-\alpha / n}$. By Proposition $4.2, L^{\varphi(\cdot)}=L^{\bar{\varphi}(\cdot)}$, so that $\bar{\varphi}^{-1}(x, s) \lesssim$ $\varphi^{-1}(x, s+h(x))$. Using this in the inequality above, and reversing the steps with $\tilde{\varphi}$, we get

$$
\left(\bar{\varphi}_{\alpha}^{\#}\right)^{-1}(x, s) \lesssim \frac{\bar{\varphi}^{-1}(x, s+h(x))}{(s+h(x))^{\alpha / n}} \lesssim \frac{\varphi^{-1}(x, s+2 h(x))}{(s+h(x))^{\alpha / n}} \lesssim\left(\varphi_{\alpha}^{\#}\right)^{-1}(x, s+2 h(x)),
$$

as required.

We are now ready for the main theorem of the paper.

Theorem 6.8. Let $\varphi \in N\left(\mathbb{R}^{n}\right)$ satisfy assumptions (AO)-(A2) and suppose that $\varepsilon>0$ is such that $t \mapsto t^{-(1+\varepsilon)} \varphi(x, t)$ is increasing and $s \mapsto s^{\frac{\varepsilon-n}{\alpha}} \varphi(x, s)$ is decreasing for every $x \in \mathbb{R}^{n}$. Then

$$
\left\|I_{\alpha} f\right\|_{\varphi_{\alpha}^{\#}(\cdot)} \lesssim\|f\|_{\varphi(\cdot)} .
$$

Note that $\varphi$ is doubling with constant $2^{\frac{n-\varepsilon}{\alpha}}$ since $s \mapsto s^{-\frac{n-\varepsilon}{\alpha}} \varphi(x, s)$ is decreasing.

Proof. Let us first note that since $s \mapsto s^{\frac{\varepsilon-n}{\alpha}} \varphi(x, s)$ is decreasing, $t \mapsto t^{-\frac{n}{\alpha}} \varphi(x, t)$ is strictly decreasing to 0 .

By Proposition 4.2 and Lemma 6.7, $L^{\varphi(\cdot)}=L^{\bar{\varphi}(\cdot)}$ and $L^{\varphi_{\alpha}^{\#}(\cdot)}=L^{\bar{\varphi}_{\alpha}^{\#}(\cdot)}$ with comparable norms. Thus it suffices to show that $\left\|I_{\alpha} f\right\|_{\bar{\varphi}_{\alpha}^{\#}(\cdot)} \lesssim\|f\|_{\bar{\varphi}(\cdot) \text {. }}$

By Propositions 4.5 and 5.1, $\bar{\varphi} \in N_{1}\left(\mathbb{R}^{n}\right)$ is normalized. By Corollary 4.4, $M$ : $L^{\bar{\varphi}(\cdot)}\left(\mathbb{R}^{n}\right) \rightarrow L^{\bar{\varphi}(\cdot)}\left(\mathbb{R}^{n}\right)$ is bounded. By Lemma 6.6, $t \mapsto t^{-\frac{n-\varepsilon}{\alpha}} \varphi(x, t)$ is almost 
decreasing. Thus, by Lemma 6.2, $\bar{\lambda}^{-1}\left(x, I_{\alpha} f(x)\right) \lesssim M f(x)$. Applying $\bar{\varphi}$ to both sides, we find that

$$
\bar{\varphi}_{\alpha}^{\#}\left(x, I_{\alpha} f(x)\right) \simeq \bar{\varphi}\left(x, \bar{\lambda}^{-1}\left(x, I_{\alpha} f(x)\right)\right) \lesssim \bar{\varphi}(x, M f(x)) .
$$

From this we deduce by the normal scaling argument that

$$
\left\|I_{\alpha} f\right\|_{\bar{\varphi}_{\alpha}^{\#}(\cdot)} \lesssim\|M f\|_{\bar{\varphi}(\cdot)} \lesssim\|f\|_{\bar{\varphi}(\cdot)} .
$$

It is well known that $|u| \lesssim I_{1}|\nabla u|$ for $u \in C_{0}^{\infty}\left(\mathbb{R}^{n}\right)$. With this we directly obtain the following result.

Corollary 6.9 (Sobolev inequality). Let $\varphi \in N\left(\mathbb{R}^{n}\right)$ satisfy assumptions (AO)-(A2) and suppose that $\varepsilon>0$ is such that $s \mapsto s^{-(1+\varepsilon)} \varphi(x, s)$ is increasing and $s \mapsto$ $s^{\frac{\varepsilon-n}{\alpha}} \psi(x, s)$ is decreasing for every $x \in \mathbb{R}^{n}$. Then

$$
\|u\|_{\varphi_{1}^{\#}(\cdot)} \lesssim\|\nabla u\|_{\varphi(\cdot)}
$$

for all $u \in C_{0}^{\infty}\left(\mathbb{R}^{n}\right)$.

If $\Omega \subset \mathbb{R}^{n}$ is a John domain, then $\left|u-u_{\Omega}\right| \lesssim I_{1}|\nabla u|$ by [5], and the same arguments yields that

$$
\left\|u-u_{\Omega}\right\|_{\varphi_{1}^{\#(\cdot)}} \lesssim\|\nabla u\|_{\varphi(\cdot)}
$$

for all $u \in W^{1,1}(\Omega)$. Here $u_{\Omega}$ denotes the average of $u$ over $\Omega$.

Let $\alpha=1$ and $\varphi(x, s)=s^{p}$ for some $p \in[1, \infty)$. Then $s \mapsto s^{-(1+\varepsilon)} \varphi(x, s)$ is increasing if $p>1$ and $s \mapsto s^{-(n-\varepsilon)} \varphi(x, s)$ is decreasing if $p<n$. Thus Theorem 6.8 and Corollary 6.9 covers the parameter range $1<p<n$ in which case $\varphi_{1}^{\#}(s)=$ $s^{p^{*}}$. The assumption $1<p$ can probably be relaxed by weak-type estimates (cf. [12, Section 6.1]), but this is left for future work.

Let $\varphi \in N$. Next we discuss the sharpness of $\varphi_{1}^{\#}$. Let $\left(r_{k}\right)$ be a positive sequence converging to zero. We set

$$
v_{k}:=r_{k} \varphi^{-1}\left(r_{k}^{-n}\right),
$$

for $k=1,2, \ldots$ Define $u_{k} \in C_{0}^{\infty}\left(B\left(0,3 r_{k}\right)\right)$ such that it equals $v_{k}$ in $B\left(0, r_{k}\right)$ and $\left|\nabla u_{k}\right| \leqslant \frac{v_{k}}{r_{k}}$. By a straightforward calculation we obtain that

$$
\int_{\mathbb{R}^{n}} \varphi\left(\left|\nabla u_{k}\right|\right) d x \lesssim r_{k}^{n} \varphi\left(\frac{v_{k}}{r_{k}}\right)=1
$$

for every $k=1,2, \ldots$ Thus $\left\|\nabla u_{k}\right\|_{\varphi} \leqslant 1$. On the other hand,

$$
\int_{\mathbb{R}^{n}} \eta\left(\left|u_{k}\right|\right) d x \gtrsim r_{k}^{n} \eta\left(v_{k}\right)=r_{k}^{n} \eta\left(r_{k} \varphi^{-1}\left(r_{k}^{-n}\right)\right)
$$

for $\eta \in \Phi$. Thus we find that the Sobolev inequality $\|u\|_{\eta} \lesssim\|\nabla u\|_{\varphi}$ does not hold for all $u \in W_{0}^{1, \varphi}(B(0,1))$ if

$$
\limsup _{t \rightarrow 0^{+}} t^{n} \eta\left(t \varphi^{-1}\left(t^{-n}\right)\right)=\infty .
$$

We consider the function $\eta:=\varphi \circ \psi^{-1}$. With the substitution $t=\varphi(r)^{-1 / n}$ we obtain

$$
\begin{aligned}
t^{n} \eta\left(t \varphi^{-1}\left(t^{-n}\right)\right) & =\frac{1}{\varphi(r)} \varphi\left(\psi^{-1}\left[\varphi(r)^{-\frac{1}{n}} \varphi^{-1}(\varphi(r))\right]\right) \\
& =\frac{1}{\varphi(r)} \varphi\left(\psi^{-1}\left[r \varphi(r)^{-\frac{1}{n}}\right]\right)=\frac{\varphi\left(\psi^{-1}[\lambda(r)]\right)}{\varphi(r)} .
\end{aligned}
$$


If the Sobolev inequality holds, then by the previous argument the limit of this expression (as $t \rightarrow 0^{+}$, i.e. $r \rightarrow \infty$ ) must be finite. Then $\frac{\varphi\left(\psi^{-1}[\lambda(r)]\right)}{\varphi(r)}<M \in[1, \infty)$ when $r>r_{0}$ for some $r_{0}$. Hence $\varphi\left(\psi^{-1}[\lambda(r)]\right)<M \varphi(r) \leqslant \varphi(M r)$ for $r>r_{0}$. Here the last inequality follows by the convexity of $\varphi$ since $M \geqslant 1$. Consequently, $\psi^{-1}[\lambda(r)] \leqslant M r$ when $r>r_{0}$ so that $\psi^{-1}(t) \leqslant M \lambda^{-1}(t)$ when $t>\lambda\left(r_{0}\right)$. If $\varphi$ is doubling, this implies that $\eta(t) \leqslant \varphi\left(M \lambda^{-1}(t)\right) \lesssim \varphi_{1}^{\#}(t)$. Hence we obtain the following proposition.

Proposition 6.10. Let $\varphi \in N$ be doubling and let $\lambda$ be as in Definition 6.5. Let $\psi:[0, \infty) \rightarrow[0, \infty)$ be such that

$$
\lim _{t \rightarrow \infty} \frac{\psi^{-1}(t)}{\lambda^{-1}(t)}=\infty
$$

Then there does not exists a constant $c>0$ such that

$$
\|u\|_{\varphi \circ \psi^{-1}} \leqslant c\|\nabla u\|_{\varphi}
$$

for all $u \in C_{0}^{\infty}(B(0,1))$.

Acknowledgement. We thank the referee for numerous minor corrections.

\section{REFERENCES}

[1] E. Acerbi and G. Mingione: Regularity results for a class of functionals with non-standard growth, Arch. Ration. Mech. Anal. 156 (2001), 121-140.

[2] M.K. Alaouia, T. Nabilab and M. Altanjia: On some new non-linear diffusion models for the image filtering, Applicable Anal. 93 (2014), no. 2, 269-280.

[3] P. Baroni, M. Colombo and G. Mingione: Non-autonomous functionals, borderline cases and related function classes. St Petersburg Mathematical Journal, to appear.

[4] P. Baroni, M. Colombo and G. Mingione: Harnack inequalities for double phase functionals. Nonlinear Anal. 121 (2015), 206-222.

[5] B. Bojarski: Remarks on Sobolev imbedding inequalities, in Complex analysis, Joensuu 1987, Lecture Notes in Math. 1351, 52-68, Springer, Berlin, 1988.

[6] Y. Chen, S. Levine, M. Rao: Variable exponent, linear growth functionals in image restoration, SIAM J. Appl. Math. 66 (2006), no. 4, 1383-1406.

[7] M. Colombo and G. Mingione: Regularity for Double Phase Variational Problems, Arch. Ration. Mech. Anal. 215 (2015), no. 2, 443-496.

[8] A. Coscia and G. Mingione: Hölder continuity of the gradient of $p(x)$-harmonic mappings, C. R. Acad. Sci. Paris, Sér. I 328 (1999), 363-368.

[9] D. Cruz-Uribe and A. Fiorenza: Variable Lebesgue spaces, Foundations and harmonic analysis, Birkhäuser/Springer, Heidelberg, 2013.

[10] D. Cruz-Uribe, A. Fiorenza, and C. Neugebauer: The maximal function on variable $L^{p}$ spaces, Ann. Acad. Sci. Fenn. Math., 28 (2003), 223-238; 29 (2004), 247-249.

[11] L. Diening: Maximal function on generalized Lebesgue spaces $L^{p(\cdot)}$, Math. Inequal. Appl. 7 (2004), 245-253.

[12] L. Diening, P. Harjulehto, P. Hästö and M. Růžička: Lebesgue and Sobolev spaces with variable exponents, Lecture Notes in Mathematics, 2017. Springer, Heidelberg, 2011.

[13] X.-L. Fan: An imbedding theorem for Musielak-Sobolev spaces, Nonlinear Anal. 75 (2012), no. 4, 1959-1971.

[14] F. Giannetti, A. Passarelli di Napoli: Regularity results for a new class of functionals with nonstandard growth conditions, J. Differential Equations 254 (2013), 1280-1305.

[15] D. Gilbarg and N. Trudinger: Elliptic partial differential equations of second order, Classics in Mathematics. Springer-Verlag, Berlin, 2001. Reprint of the 1998 edition.

[16] P. Harjulehto, P. Hästö and R. Klén: Generalized Orlicz spaces and PDE, Preprint (2015).

[17] P. Harjulehto, P. Hästö, V. Latvala and O. Toivanen: Critical variable exponent functionals in image restoration, Appl. Math. Letters 26 (2013), 56-60. 
[18] P. Harjulehto, P. Hästö, U. Lê and M. Nuortio: Overview of differential equations with nonstandard growth, Nonlinear Anal. 72 (2010), no. 12, 4551-4574.

[19] P. Hästö: The maximal operator on generalized Orlicz spaces, J. Funct. Anal 269 (2015), 40384048.

[20] P. Hästö: The maximal operator on generalized Orlicz spaces - Erratum, preprint (2015).

[21] P. Hästö, Y. Mizuta, T. Ohno and T. Shimomura: Sobolev inequalities for Orlicz spaces of two variable exponents, Glasgow Math. J. 52 (2010), 227-240.

[22] F.-Y. Maeda, Y. Mizuta, T. Ohno and T. Shimomura: Boundedness of maximal operators and Sobolev's inequality on Musielak-Orlicz-Morrey spaces, Bull. Sci. Math. 137 (2013), 76-96.

[23] F.-Y. Maeda, Y. Mizuta, T. Ohno and T. Shimomura: Approximate identities and Young type inequalities in Musielak-Orlicz spaces, Czechoslovak Math. J. 63(138) (2013), no. 4, 933-948.

[24] G. Mingione: Regularity of minima: an invitation to the dark side of the Calculus of Variations, Appl. Math. 51 (2006), 355-426.

[25] Y. Mizuta, T. Ohno and T. Shimomura: Sobolev's inequalities and vanishing integrability for Riesz potentials of functions in the generalized Lebesgue space $L^{p(\cdot)} \log L^{q(\cdot)}$, J. Math. Anal. Appl. 345 (2008), 70-85.

[26] J. Musielak: Orlicz spaces and modular spaces, Lecture Notes in Mathematics, 1034. Springer, Berlin, 1983.

[27] A. Nekvinda: Hardy-Littlewood maximal operator on $L^{p(x)}\left(\mathbb{R}^{n}\right)$, Math. Inequal. Appl. 7 (2004), 255-266.

[28] T. Ohno and T. Shimomura: Trudinger's inequality for Riesz potentials of functions in MusielakOrlicz spaces, Bull. Sci. Math. 138 (2014), no. 2, 225-235.

[29] W. Orlicz: Über konjugierte Exponentenfolgen, Studia Math. 3 (1931), 200-211.

[30] A. Świerczewska-Gwiazda: Nonlinear parabolic problems in Musielak-Orlicz spaces, Nonlinear Anal. 98 (2014), 48-65.

[31] A. Wróblewska-Kamińska: Existence result for the motion of several rigid bodies in an incompressible non-Newtonian fluid with growth conditions in Orlicz spaces, Nonlinearity 27 (2014) $685-716$.

\section{P. HARJUlEhTO AND P. HÄSTÖ}

Department of Mathematics and Statistics, FI-20014 University of Turku, Finland

petteri.harjulehto@utu.fi, peter.hasto@oulu.fi

P. HÄSTÖ

Department of Mathematical Sciences, FI-90014 University of Oulu, Finland 